INRA Prod. Anim., 2017, 30 (3), 229-240

\section{Perception et attitude des consommateurs vis-à-vis des produits de terroir : implications pour les fromages}

\author{
P. ZINDY', R. HALAWANY-DARSON ${ }^{2,3}$, A. HAUWUY' \\ CNAOL - 42 rue de Châteaudun, 75314, Paris, France \\ 2 VetAgro Sup, 89 avenue de l'Europe, 63370, Lempdes, France \\ ${ }^{3}$ Université Clermont Auvergne, INRA, UMRF, 15000, Aurillac, France \\ ${ }^{4}$ CERAQ, 40 rue du Terraillet, 73190, Saint Badolphe, France \\ Courriel : rafia.halawany-darson@vetagro-sup.fr
}

\begin{abstract}
Dans l'offre agro-alimentaire proposée aux consommateurs, la mention de l'origine d'un produit et en particulier la référence à des attributs du « terroir » constituent de puissants leviers de segmentation et de positionnement. Cet état de l'art présente une analyse des mécanismes sous-jacents à la perception des produits de terroir par les consommateurs et apporte ainsi un éclairage essentiel aux acteurs économiques en charge de l'organisation de l'offre.
\end{abstract}

Le marché des produits alimentaires perçus comme « de terroir » est porteur. Le terme terroir lui-même fait l'objet de plusieurs études et fait couler beaucoup d'encre dans différents pays (Bowen et Mutersbaugh 2014). Il connaît un intérêt toujours croissant des consommateurs et trouve écho à la fois dans des initiatives politiques visant à développer la revendication d'origine sur les produits (reconnaissance des Indications Géographiques au niveau communautaire en 1992, émergences plus récentes des marques collectives territoriales) et dans les initiatives des opérateurs des filières agro-alimentaires et des distributeurs pour développer l'offre perçue comme " terroir ». Cet engouement de « patrimonialisation » amorcé dès les années 1970 (Guillaume 1980) est toujours d'actualité auprès de consommateurs désireux d'un « retour aux racines » (Amilien 2005, AFP 2014). L'industrialisation de la sphère alimentaire génère chez le consommateur des angoisses qui appellent une symbolique d'enracinement rural et affectif (Poulain 1993). Il s'agit là d'une des caractéristiques du consommateur hypermoderne (Aubert 2006) qui est nostalgique du passé et à la recherche de produits authentiques. Cette demande offre ainsi aux opérateurs agro-alimentaires un levier puissant de différenciation et de création de valeur (Fort et Fort 2006). Les productions localisées qui présentent une grande diversité en France sont dès lors convoitées dans la mesure où la mise en avant de leur origine peut apporter une plusvalue économique (Bérard et Marchenay 2004). Avec à la clé des stratégies dédiées, même les marques de distributeurs redou- blent d'effort en misant sur le terroir pour améliorer leur attractivité (AFP 2015).

Par rapport à cet engouement, les filières fromagères valorisant leur terroir sont interrogatives quant au positionnement de leurs produits : quelle est l'image des fromages de terroir pour les consommateurs ? Quelle perception en ont-ils ? Quels sont les attributs des produits que les consommateurs perçoivent? Quel est le lien entre cette perception et les comportements d'achat? Est-ce que les stratégies marketing des filières fromagères valorisant leur terroir sont adaptées?

En effet, les connaissances des filières de terroir sur les consommateurs sont souvent tirées des études de marché et des baromètres et peu portées sur la demande et la compréhension approfondie des motivations et comportements des consommateurs. Il en résulte une conceptualisation des produits de terroir le plus souvent issue de la vision qu'en ont les producteurs et les groupements de coordination de l'offre (Aurier et al 2004). Le champ de production du concept «terroir" reste également celui des chercheurs et des scientifiques et c'est cette approche qui circule dans le champ des connaissances utilisées par les producteurs, le législateur et les consommateurs (Fort et Rastoin 2009). De fait, cette approche ne répond pas à toutes les interrogations. Par ailleurs, les études sur la perception des produits de terroirs par les consommateurs sont dispersées et pas toujours portées à connaissance des structures en lien avec la promotion de ces produits.
Aussi, cette présente revue de littérature s'inscrit dans un double objectif: synthétiser les travaux réalisés sur la perception des produits de terroir et porter ces résultats à connaissances des opérateurs économiques de ces filières. Dans cette perspective, cette revue propose dans un premier temps un rappel du cadre entendu par « produit du terroir » ainsi que leur importance économique. Une seconde partie présente la perception des produits de terroir par les consommateurs et les effets des sources perçues du terroir sur l'attitude et la préférence des consommateurs vis-à-vis de ces produits. Enfin, la troisième partie propose des pistes d'application de ces connaissances en communication et stratégie marketing en essayant de dégager des leviers d'action. Une analyse critique conclut cette partie en présentant les pistes à creuser et les questions restant en suspens.

\section{1 / Produit de terroir : défi- nition et cadre conceptuel}

\section{1 / Conceptualisation du terroir}

a) D'un point de vue académique

Il n'existe pas de définition consensuelle du concept « produit de terroir» (Aurier et al 2004). Les auteurs ayant travaillé à la définition du concept « terroir » sont nombreux. La littérature souligne une interprétation ambigüe (Bérard et Marchenay 1995, Kreziak et Lenglet 2009) et complexe (Beylier et al 2010) qui renvoie à une vaste catégorie fourre- 
tout (Bérard et Marchenay 2004) ou un « tout», dans lequel le lieu agit comme révélateur (Amilien 2005). Concept polysémique, s'il fallait présenter une définition du terroir, celle proposée par Beylier et al (2010) issue d'une revue de littérature et fortement inspirée par la définition développée par Casabianca et al (2008) semble intéressante à présenter ici car complète en termes de champs disciplinaires : "Le terroir peut être considéré comme un espace territorial délimité, vivant et innovant dans lequel une communauté humaine construit, au cours de son histoire, un savoir collectif de production fondé sur un système d'interactions entre un milieu physique et biologique, et un ensemble de facteurs humains, et dont les valeurs patrimoniales sont les fruits de relations complexes et de longue durée entre des caractéristiques culturelles, sociales, écologiques et économiques. Les savoir-faire ainsi mis en jeu révèlent une originalité, confèrent une typicité et aboutissent à une réputation pour un bien originaire de cet espace géographique. Ces savoir-agir en lien avec l'habitat naturel façonnent le paysage, préservent la biodiversité, les diversités sociales et culturelles en conformité avec les objectifs de développement durable.»

La majorité des auteurs s'accorde sur l'existence de deux dimensions intimement liées dans un produit alimentaire de terroir : une dimension territoriale (facteurs naturels - espace : ancrage d'un produit dans un lieu géographique, sol, climat, environnement global) et une dimension culturelle et historique (facteurs humains - temps et culture : histoire, savoir-faire, notoriété) (Aurier et al 2004, Fort et Fort 2006, Lenglet 2011). Un produit peut satisfaire à des degrés divers ces dimensions et présenter en conséquence un ancrage " terroir » plus ou moins fort du point de vue du consommateur (Fort et Fort 2006). Ces deux dimensions peuvent ainsi servir pour réaliser un premier tri dans l'univers des produits de terroir, entre produits qui font sens et produits opportunistes (Bérard et Marchenay 2004).

\section{b) D'un point de vue règlementaire}

Plusieurs signes officiels de qualité et d'origine (SIQO) liés au produit, recoupent à la fois une dimension territoriale et une dimension culturelle et historique pouvant faire correspondre le produit à un produit de terroir. C'est le cas de l'AOP (Appellation d'Origine Protégée) et de l'IGP (Indication Géographique Protégée). Dans le cas de l'AOP, la validation d'une relation au terroir par une démarche d'identification de ce qui fait l'aspect traditionnel avec une approche d'expertise scientifique sont une condition requise pour obtenir cette reconnaissance (Bérard et Marchenay 1995). L'IGP exige la même approche mais avec un ancrage au terroir qui peut être limité à une des étapes d'élaboration du produit.

D'autres signes de qualité existent tels que l'agriculture biologique, le label rouge ou la STG (Spécialité Traditionnelle Garantie), cependant le critère territorial est absent de l'obtention de ces labels officiels. Quant à la mention valorisante " produit de montagne » (définie par le règlement UE 1151/2012), elle exige un ancrage géographique mais ne requiert pas de dimension culturelle et historique.

Les signes officiels AOP ou IGP ne sont toutefois pas les seules possibilités pour rattacher un produit à un terroir. Plusieurs initiatives autres que les SIQO restent à portée des entreprises : produits locaux, produits typiques, produits authentiques, produits régionaux, produits fermiers et autres mélanges de termes rappelant la notion de territoire ou de localité liée à la culture de l'alimentation, marques collectives, marques privées, etc. (cf. encadré 1). À partir du moment où les opérateurs respectent le code de la consommation et notamment les principes de véracité (l'information ne doit pas induire en erreur le consommateur) et de disponibilité (le nom du produit ne doit pas être protégé par une AOP ou une IGP), il n'y a pas de disposition qui restreigne le rattachement d'un produit au terroir (Fort et Rastoin
2009). Il faut finalement retenir que le terroir peut être un objet activé par une marque, un signe de qualité ou un packaging (Beylier et al 2010).

Au milieu de cette diversité sémantique et règlementaire, cette revue de littérature s'attachera à prendre en compte toutes les études pertinentes sur la question de perception des produits de terroir. C'est-à-dire un produit recoupant une dimension territoriale ainsi qu'une dimension culturelle et historique, que ces dimensions soient portées par une marque, des informations sur le packaging, une IG ou un recoupement de ces éléments.

\section{2 / Importance économique des produits de terroir}

Les données les plus facilement mobilisables et les plus précises concernent les SIQO. Les données de marché sur ces produits sont en effet nombreuses et détaillées. Ainsi en 2010, les ventes en valeur des produits sous Indication Géographiques (IG) (AOP et IGP) dans l'Union européenne des 27 États membres étaient estimées à 54,3 milliards d' $€$. Plus de la moitié $(56 \%)$ de ces ventes sont liées aux vins, $29 \%$ sont liées aux produits agro-alimentaires et $15 \%$ aux spiritueux (tableau 1, Chever et al 2012). En France, 22\% des exploitations agricoles disposent d'au moins une production sous signe de qualité, hors produits viticoles et biologiques. Les SIQO concernent plus de 1100 produits. En 2014, le chiffre d'affaires à la première mise en marché des produits sous signe d'identification de la qualité et de l'origine (SIQO) hors bio est estimé à environ 22 milliards d'euros HT, dont un peu moins de 16 milliards pour les vins sous SIQO (INAO 2014).

Ces chiffres sous-estiment toutefois les ventes réalisées sur l'ensemble des produits marquetés " terroir », cette catégorie étant vaste et mal définie. Un indicateur utilisé dans la littérature concerne le nombre de marques commerciales déposées à l'Institut National

Tableau 1. Ventes en valeurs (M€) par filières sous Indications Géographiques entre 2005 et 2010 dans I'UE des 27. (Source : Chever et al 2012).

\begin{tabular}{|l|r|r|r|r|r|r|c|}
\hline & $\mathbf{2 0 0 5}$ & $\mathbf{2 0 0 6}$ & $\mathbf{2 0 0 7}$ & $\mathbf{2 0 0 8}$ & $\mathbf{2 0 0 9}$ & $\mathbf{2 0 1 0}$ & $\mathbf{\%} \mathbf{2 0 1 0}$ \\
\hline Vins & 27963 & $\mathbf{2 8 9 0 0}$ & $\mathbf{3 0 8 8 4}$ & 31363 & 29025 & 30376 & 55,9 \\
\hline $\begin{array}{l}\text { Produits agricoles et } \\
\text { denrées alimentaires }\end{array}$ & 13284 & 13457 & 13891 & 14238 & 14525 & 15790 & 29,1 \\
\hline Spiritueux & 7168 & 7555 & 8101 & 7793 & 7126 & 8149 & 15,0 \\
\hline Vins aromatisés & 31 & 31 & 31 & 31 & 31 & 31 & 0,1 \\
\hline Total & $\mathbf{4 8} \mathbf{4 4 6}$ & $\mathbf{4 9} \mathbf{9 4 3}$ & $\mathbf{5 2 9 0 7}$ & $\mathbf{5 3} \mathbf{4 2 5}$ & $\mathbf{5 0 7 0 7}$ & $\mathbf{5 4 3 4 6}$ & $\mathbf{1 0 0}$ \\
\hline
\end{tabular}


Encadré 1. Catégorisation des produits pouvant être perçus comme « de terroir ».

Cet encadré regroupe les catégorisations trouvées dans la littérature et pouvant recouper les deux dimensions (territoriale et culturelle) constitutives des produits de terroirs :

Produits fermiers. Ces derniers sont fréquemment assimilés à des produits de terroir. Toutefois, s'ils ne répondent pas à une production inscrite dans une dimension culturelle locale, ces derniers n'ont pas d'autre particularité que d'être produits à l'échelle de l'exploitation à partir des matières premières produites sur place (Berard et Marchenay 2004). En ce sens, ils répondent à la dimension territoriale (facteurs naturel, origine) mais peuvent ne pas répondre à la dimension culturelle et/ou historique (culture partagée).

Produits locaux, de proximité. Les travaux concernant les produits locaux sont intéressants à rapprocher du cadre de cette étude. Ces produits constituent en effet l'un des secteurs les plus dynamiques de la consommation alimentaire ces dernières années en Europe (Amilien 2005). Ils représenteraient une offre de 7 à 10 milliards d'€ en France (Delvalée 2015), avec également des croissances fortes aux États-Unis (8 100 marchés locaux recensés, secteur de 7 milliards de \$). De la même manière qu'un produit fermier, un produit local peut avoir comme seule particularité une production de proximité ou au contraire s'inscrire dans un ancrage terroir plus prononcé s'il est en lien avec une dimension culturelle locale. En effet, le terme « local » correspond à un terme général contenant plusieurs dimensions différentes et complémentaires : i) un lieu qui souligne souvent les caractéristiques historiques, culturelles et sociales et ii) un espace physique, territoire, dont le produit tire sa typicité et ses caractéristiques (Holt et Amilien 2007). Dans cette interprétation large des produits locaux, ces derniers recoupent bien les dimensions des produits de terroir. Van der Meulen (2007) propose un outil méthodologique mettant en valeur les contributions de quatre différents facteurs (territorialité, typicité, tradition, pratique collective) au degré de connectivité des produits alimentaires à leur lieu d'origine.

Productions localisées. Berard et Marchenay (2004) avancent la notion de productions localisées. Pour les auteurs, deux paramètres permettent d'englober l'ensemble de ces productions liées à un lieu : la profondeur historique et les savoir-faire partagés. Ainsi, leur inscription en un lieu s'assortit d'une antériorité et de pratiques collectives. Ce sont ces deux aspects qui permettent de distinguer la provenance (venir d'un lieu), de l'origine (être de ce lieu). Pour les auteurs, la notion de productions localisées permet ainsi de faire un tri plus fin au sein des « produits de terroir » qui peuvent confondre origine et provenance.

Produits régionaux. Un produit régional est défini comme un produit dont la qualité et/ou la renommée peut être attribuée à sa région d'origine. II est de plus marqueté en utilisation du nom de cette région d'origine (Van Ittersum 2007).

Produits typiques. Un produit typique se distingue d'un produit industrialisé standard par ses caractéristiques sensorielles, émotionnelles et sanitaires (Delfosse 1991). De plus, Letablier et Nicolas (1994) notent que ce type de produit est associé à une représentation de goût et de qualité qui évoque une tradition, un mode de vie et une forme d'authenticité.

Produits artisanaux. Pour cette catégorie, c'est le rattachement à un savoir-faire collectif qui peut limiter le rattachement systématique de ces produits au terme " produit de terroir ». Une spécialité développée par un artisan peut être issue de produits locaux, voir bénéficier d'une antériorité importante mais si elle est issue d'une initiative individuelle, elle ne recoupe pas la définition de produit de terroir entendue ici, du fait de l'absence d'une culture partagée. C'est le cas par exemple des confiseurs, des pâtissiers... Par ailleurs pour les produits artisanaux, le focus est souvent mis sur le processus technique plutôt que sur l'origine de la matière première (Van der Meulen 2007).

Produits authentiques. Notion complexe puisque polymorphe (Taylor 2001), les dimensions d'un produit perçu comme authentique sont au nombre de trois : i) l'origine du produit, ii) sa singularité et la iii) projection des valeurs du consommateur dans le produit (Camus 2004). L'authenticité va de pair avec la quête de nostalgie des produits naturels par les consommateurs qui veulent un retour à la nature et aux saveurs d'antan. Selon Rosselin (1994), Brown et al (2003) pensent que « la quête d'authenticité est l'une des pierres angulaires du marketing contemporain ».

Produits naturels. Un produit naturel est défini par opposition au produit transformé (Thévenot, 2007 in Salvador 2011). C'est un produit qui a subi le moins de transformations possibles même si le caractère naturel concerne surtout sa composition et moins sa fabrication.

Produits traditionnels. Un produit alimentaire traditionnel est un « produit consommé fréquemment ou associé à des fêtes et/ou saisons spécifiques, transmis de générations en générations, fait d'une manière spécifique selon un patrimoine gastronomique, préparé de manière naturelle, reconnu en raison de ses propriétés sensorielles et associé à un certain lieu, région ou pays » (Vanhonacker et al 2010). Cette définition globale est issue d'une étude quantitative pan-européenne sur le point de vue des consommateurs. Un produit traditionnel recoupe de ce fait une dimension territoriale et culturelle et rejoint de ce fait la notion de produit de terroir.

Système agroalimentaires localisés (SYAL). Les SYAL sont définis comme des ensembles " d'organisations de production et de service (unités de production agricole, entreprises agroalimentaires, commerciales, de services, restauration) associées à un territoire spécifique par leurs caractéristiques et leur fonctionnement. Le milieu, les produits, les hommes, leurs institutions, leur savoir-faire, leurs comportements alimentaires, leurs réseaux de relations, se combinent dans un territoire pour produire une forme d'organisation agroalimentaire à une échelle spatiale donnée » (CIRAD-SAR 1996). 
de la Propriété Intellectuelle et portant le terme « terroir ». Il en est enregistré un peu plus de 1011 au $1^{\text {er }}$ trimestre 2016, contre 884 en 2004 (Rastoin 2004). Le pourcentage des marques déposées dans le secteur agroalimentaire et utilisant le préfixe ou le suffixe « terroir » est passé de $30 \%$ à $62 \%$ entre 1990 et 2000 . Cette dynamique a également été appuyée par les distributeurs avec leurs marques dédiées « terroir » telles que Reflets de France (Carrefour), Nos régions ont du talent (Leclerc) et Terres et saveurs (Casino) (Aurier et Fort 2005). Fort et Rastoin (2009) ont établi une typologie de la segmentation du marché agroalimentaire en distinguant le marché des produits différenciés par leur typicité géographique (produits de «terroir » ou produits «gourmands»). Ils estimaient ainsi en 2004 un chiffre d'affaires de 26 milliards d'€ pour ce dernier segment sur un total de 129 milliards destinés à l'alimentation en France.

\section{2 / Perception des produits de terroir par les consommateurs}

Les définitions des principaux concepts mobilisés dans cette partie sont développées dans l'encadré 2 .

\section{1 / Des produits de terroir forte- ment évocateurs}

Les aliments ne sont pas des objets ordinaires : nous les incorporons et, ce faisant, nous incorporons par analogie les qualités réelles ou imaginaires, positives ou négatives, que nous leur prêtons (principe de l'incorporation-Fischler 1990). Du point de vue des consommateurs, les produits de terroir représentent une charge affective très forte et cristallisent des attentes aussi diverses que le goût, la santé, la protection de l'environnement, le soutien aux petits producteurs (Fort et Fort 2006). Le consommateur se représente une vision idéalisée où le terroir, comme l'origine est lié à de nombreuses valeurs ajoutées comme la tradition, la nature mais à un niveau imaginaire ou à travers une « réalité empruntée » ou « hyper réalité » (Amilien et al 2007). Fort et Fort (2006) parlent ainsi de monde mythique où l'achat d'un produit de terroir procurerait le droit de s'immiscer dans un système autre à son propre système socio-culturel local. La quête de l'authentique mène ainsi les consommateurs à «fantasmer des produits » qui n'ont pas forcément beaucoup de rapport avec la réalité historique.

Ces évocations riches ressortent également dans les études consommateurs réalisées par les groupements de producteurs. Pour ce qui est du lait, une étude d'image qualitative réalisée par BVA
(Nicolas et Rainsard 2005) sur le lait de brebis qui fait ressortir des imaginaires très développés et assez fortement différenciés selon les types de lait. Une capacité d'évocation forte pour l'univers normand est également ressortie d'une étude Eurofins qualitative et quantitative menée en Basse-Normandie (Dhaene et Theet 2010). Plus récemment, l'étude qualitative « usages et attitudes de consommation de fromages » réalisée en 2011 par A\&S pour le CNIEL (Demarty 2010) fait également ressortir spontanément une famille des fromages « authentique/terroir » très valorisée et très évocatrice quels que soient les groupes cibles. Les dimensions goût, authenticité, région, culture, savoir-faire, naturalité ressortent comme caractéristiques de cette famille.

\section{2 / Les sources perçues du terroir par les consommateurs}

Tel que vu dans la partie « conceptualisation du terroir », la catégorie «terroir » a principalement été définie d'un point de vue de l'offre par les filières. Des travaux plus récents se sont attachés à étudier les sources perçues des produits de terroir par le consommateur, c'est-àdire les critères nécessaires de son point de vue pour qu'un produit soit catégorisé « terroir ». Les travaux d'Aurier et al (2004) confortés par Lenglet (2011) ont mis en évidence trois sources perçues du terroir par les consommateurs :

i) Métier (regroupant savoir-faire, recette et tradition);

ii) Temps et culture (regroupant histoire et rituel);

iii) Origine géographique (regroupant territoire, région et terre).

Les auteurs ont montré que le facteur « temps et culture » était le mieux lié à l'ensemble des associations, évocations et représentations associées au fait qu'un produit soit de terroir. C'est par ailleurs le seul corrélé avec la qualité perçue : la qualité perçue est ainsi plus ancrée dans la référence au passé que dans la zone géographique ou le mode de fabrication, confirmant des résultats de travaux antérieurs (Steenkamp 1990, Aurier et al 2004). Le facteur " origine » serait au contraire le seul à avoir un effet significatif sur l'attitude (Aurier et al 2004).

Une approche complémentaire intéressante à présenter pour décrire les sources perçues du terroir est celle présentée dans l'article de Beylier et al (2010), citant Dubois et al (2001) qui met en parallèle les produits de terroir avec une approche perceptuelle du luxe. Ces produits disposent d'au moins six caractéristiques importantes pour le consommateur dont un prix élevé conformément à la qualité garantie ; la disposition à payer de consommateurs « terroir » étant 1,7 fois supérieure à celle des autres. Cette valeur est proche du différentiel de prix observé via les panels entre fromages AOP, en moyenne 1,65 fois plus chers que les fromages non AOP (INAO et CNAOL 2015), bien que la catégorie non AOP intègre également des fromages marquetés terroir.

Les produits de terroir, étant associés à des attributs de qualité uniques, sont bien souvent plus chers que ceux sans l'appellation terroir (Deselnicu et al 2013), cependant, la propension à payer pour de tels produits ne serait présente que si les consommateurs comprennent la valeur ajoutée de ces produits. Cette dernière est liée à un lieu géographique, à un savoir-faire, à un héritage et à un goût particulier. De façon plus générale, la propension à payer pour les produits alimentaires avec IG varie de façon significative en fonction des produits et des marchés (Deselnicu et al 2013). Concernant les produits laitiers, les consommateurs européens ont en moyenne une propension à payer positive, par contre, il existe dans la littérature des résultats contradictoires concernant le fromage (Ngoulma 2015).

\section{3 / Le pays d'origine ou la région d'origine, des attributs essentiels de la signalisation de la qualité}

L'effet du pays ou de la région d'origine est un courant de littérature souvent sollicité pour éclairer l'étude des produits de terroir puisqu'il s'intéresse au rôle de l'origine géographique dans l'évaluation des produits. Informer le consommateur de l'origine du produit via l'étiquetage est motivé par la reconnaissance que l'image et la notoriété de la région sont souvent corrélées à la qualité globale d'un produit (Menapace et al 2009).

L'origine du produit est généralement considérée comme un attribut extrinsèque à l'objet, un signal conféré par le pays ou la région de rattachement. L'origine agit comme une information qui touche d'autres caractéristiques du produit et peut affecter directement la valeur de l'alimentation par son rôle symbolique ou affectif (Verlegh et Steenkamp 1999, Stefani et al 2006). L'origine apporte des informations utilisées par le consommateur pour inférer des croyances quant à sa qualité. En ce sens, l'association du nom de la région d'origine au nom du produit peut être comparée à une stratégie de marque (Van der Lans 2001, Van Ittersum et al 2003). L'importance de l'origine et son effet significatif sur l'évaluation du consommateur a été soulignée à de nombreuses reprises (Aurier et Fort 2005, Dekhili et D'Hauteville 2006). L'influence du pays d'origine d'un pro- 
Encadré 2. Définitions des concepts.

Perception : La perception correspond au processus par lequel un individu choisi, organise et interprète des éléments d'information externes pour construire une image cohérente de son environnement (Kotler et Dubois 2003). II s'agit d'un processus subjectif, sélectif et cumulatif.

\section{Signaux intrinsèques, Signaux extrinsèques :}

- Intrinsèques : signaux liés aux attributs des produits qui altèrent les propriétés physiques du produit. Leur modification n'est possible que par une modification du produit. Ils sont liés à la composition physique du produit et cessent d'exister une fois le produit consommé (Verdù Jover et al 2004).

- Extrinsèques : signaux liés aux attributs qui ne font pas partie du produit même et dont la modification n'affecte pas la composition de celui-ci. II peut s'agir du prix, de l'emballage, de normes, d'un label ou SIQO, de marque, d'une origine géographique, d'allégations, d'une réputation, etc. Les attributs extrinsèques peuvent se substituer à un manque d'information sur les attributs intrinsèques. Ils représentent des variables d'action marketing potentielles.

Pour évaluer la qualité d'un produit, les consommateurs utilisent ces deux catégories de signaux (Jacoby et al 1971, Simonson 1989). L'utilisation des caractéristiques extrinsèques est plus fréquente lorsque la qualité du produit n'est pas directement observable et qu'elle n'est identifiable qu'après consommation (Nelson 1970, Richardson et al 1994). Les signaux extrinsèques sont plus facilement identifiés et interprétés par les consommateurs, ils ont ainsi une valeur de confiance élevée alors que les signaux intrinsèques eux, bénéficient d'une valeur de prédiction élevée (Richardson et al 1994 et Blair et Innis 1996 in Halawany-Darson 2010)

Qualité perçue : Définie par Steenkamp (1986), elle sert d'intermédiaire entre la qualité objective du produit et les préférences des consommateurs. La qualité perçue correspond à l'évaluation ou jugement global de la qualité par le consommateur et est le résultat de la qualité attendue et de la qualité expérimentée (Verdù Jover et al 2004). La qualité perçue est spécifique au produit considéré (Steenkamp 1986).

Préférences : Les préférences sont l'expression d'un choix, en raison de critères soit subjectifs, soit objectifs. Elles sont le croisement entre les croyances de qualité et le désir de qualité qui forme la base des préférences du consommateur (Van der Lans 2001). Dans le domaine alimentaire, le rôle de l'expérience comme fondement des préférences est particulièrement développé (Sirieix 1999). Les études consommateurs soulignent toute la complexité des facteurs affectant les préférences en termes de nourriture (y compris les facteurs environnementaux, sociaux et psychologiques, cf. § 3.3). La préférence d'un produit est fonction de la perception des attributs du produit, qui peut elle-même être influencée (Aurier et al 2004). Notamment, la connaissance et le niveau d'information jouent un rôle important dans la préférence. Au sens économique du terme, la littérature distingue les "préférences déclarées », utiles lorsque les comportements ne peuvent être observés (par exemple lorsque les marchés n'existent pas), des « préférences relevées » déduites des choix des consommateurs.

Attitude : L'attitude correspond à la « disposition à réagir de façon favorable ou défavorable à un objet particulier ou à une classe d'objets » et découle des motivations alimentaires, c'est-à-dire de l'ensemble des considérations susceptibles d'être prises en compte par les individus pour choisir ce qu'ils vont manger (Steptoe et al 1995). L'attitude est considérée comme l'un des meilleurs prédicteurs du comportement de consommation par la majorité des modèles d'analyse en raison de sa capacité reconnue à appréhender l'évaluation globale qui est faite par un consommateur (Darpy 2012), l'évaluation d'un produit étant fonction de l'attitude pour le produit (Aurier et Fort 2005). On considère généralement trois composantes de l'attitude (Rosenberg et Hovland 1960):

i) Une composante cognitive (croyances et connaissances relatives à l'objet considéré) ;

ii) une composante affective (jugement global et affectif, attentes, préférences) ;

iii) une composante conative (intention de comportements et comportements effectifs). Inscrite dans une approche plus économique, elle est notamment appréhendée via l'intention d'achat et la mesure du consentement à payer.

Les trois composantes de l'attitude s'articulent en fonction du niveau d'implication du consommateur, la nature du produit et le type de situation. Celsi et Olson (1988) proposent que l'implication du consommateur influence le montant et la direction de son attention, son processus de compréhension et l'effort cognitif et physique effectué durant le processus de compréhension. Le processus décisionnel peut être plus ou moins long en fonction de l'implication. Sherif et Cantril (1947), définissent l'implication comme " un état de tension qui naît chez l'individu à partir du lien entre ses valeurs fondamentales et une situation ». La façon dont un individu réagit dans une situation donnée ou un problème dépend donc de son implication, celle-ci étant fonction de l'intérêt qu'il porte à un produit ou à une situation (Halawany-Darson 2010). L'attitude peut finalement être définie comme le degré selon lequel un individu aime ou n'aime pas un produit, l'apprécie ou non et y est favorable ou non (Beylier et al 2010).

duit sur les évaluations des consommateurs dépend de plusieurs critères : le degré perçu de la pertinence de cette information, la motivation, l'implication, la préférence pour un pays particulier, le risque perçu, le nombre d'attributs, l'importance de l'origine en ce qui concerne les autres éléments, et la facilité de trouver l'information. Obermiller et Spangenberg (1989) distinguent les aspects cognitifs (croyances envers l'origine), affectifs (sentiments, émotions) et normatifs (considération morale -ethnocentrisme, boycott) qui sous-tendent l'effet de l'ori- gine géographique sur la prise de décision des consommateurs. La perception du consommateur est influencée par sa proximité avec le produit. On observe une proximité cognitive liée à une proximité géographique mais à la condition que le consommateur identifie son lieu 
de résidence à celui de production du produit (Olivier et al 2014).

Il faut toutefois tenir compte du fait, qu'en tant qu'attribut parmi d'autres, l'origine d'un produit ne suffit pas à elle seule à transmettre une vraie authenticité au consommateur. D'autant qu'avec la globalisation, l'image de l'origine est devenue plus complexe. L'origine du produit se compose du pays associé au produit, le pays de la fabrication et le pays de la conception. Aussi, les consommateurs déduisent cette authenticité, cette « régionalité » via un ensemble d'informations : les attributs physiques du produit, le lieu d'achat ou de consommation, les éléments de patrimoine communiqués, etc. (Treager et al 1998).

\section{3 / Les déterminants et facteurs explicatifs des perceptions}

\section{1 / Effet de la région d'origine sur la qualité perçue, l'attitude et la préférence du produit}

En tant qu'attribut essentiel de signalisation de la qualité, l'origine du produit a fait l'objet de nombreux travaux pour mieux comprendre la manière dont cette information agit sur le consomma- teur. La figure 1 (Influence de la région d'origine (image et attitude) sur la préférence du produit) synthétise les principaux enseignements de ces travaux.

L'influence du pays d'origine est généralement appréhendée à travers l'image région/pays d'origine qui influencerait l'évaluation du produit par un effet de halo issu des associations existantes que les consommateurs ont de cette région/ pays. Cette image régionale du produit spécifique constitue l'ensemble des croyances que les consommateurs ont sur l'aptitude de la région pour la production de ce produit (Van Ittersum et al 2003). L'image région d'origine est d'une nature essentiellement cognitive (Aurier et Fort 2005, Bernard et Zarrouk-Karoui 2013) et son effet sur la préférence d'un produit est spécifique au produit considéré (Van Ittersum et al 2003). Ainsi, l'effet «Savoie » ne sera pas le même sur un fromage que sur un produit manufacturé par exemple. C'est ce que les auteurs Haübl et Elrod (1999) ont appelé congruence entre le produit et le pays d'origine : le consommateur perçoit la spécialisation des terroirs, leur typicité et leur capacité à fabriquer le produit (Aurier et al 2004) avec pour conséquence une image régionale transmise plus facilement au produit et avec un effet plus fort. Chamart et Liquet (2009) notent ainsi que les terroirs présentent des disparités fortes de pouvoirs d'évocation, certain ayant des potentiels supérieurs aux autres. Aussi, l'origine des fromages d'Auvergne explique certainement pour partie l'image forte qu'a le consommateur de ces produits puisque 93\% des acheteurs de fromages pensent que les fromages d'Auvergne reflètent bien la nature de l'Auvergne (AFA 2010). Par ailleurs, plus l'adéquation entre la région et le produit est forte, plus l'effet pays d'origine sera puissant (Van Ittersum et al 2003). De même, plus l'aire du lieu d'origine est restreinte, plus l'attente du consommateur en termes de qualité est élevée (Stefani et al 2006), une région étant plus homogène qu'un pays.

En plus de l'image région/pays d'origine, d'autres travaux ont précisé l'influence de l'attitude envers la région d'origine sur la préférence d'un produit (Van Ittersum et al 2003, Dekhili et D'Hauteville 2006, Bernard et Zarrouk-Karoui 2013). Il est à préciser que l'ensemble des images, et des représentations associées à la région d'origine du produit alimentaire influence les préférences du consommateur envers ces produits.

Dans le cas spécifique de produits de terroir, l'attitude d'un consommateur envers un pays ou une région est fonction de trois composantes complémentaires :

Figure 1. Influence de la région d'origine (image et attitude) sur la préférence du produit (d'après Van Ittersum et al 2003, Aurier et al 2004, Dekhili et D'Hauteville 2006, Van Ittersum et al 2007).

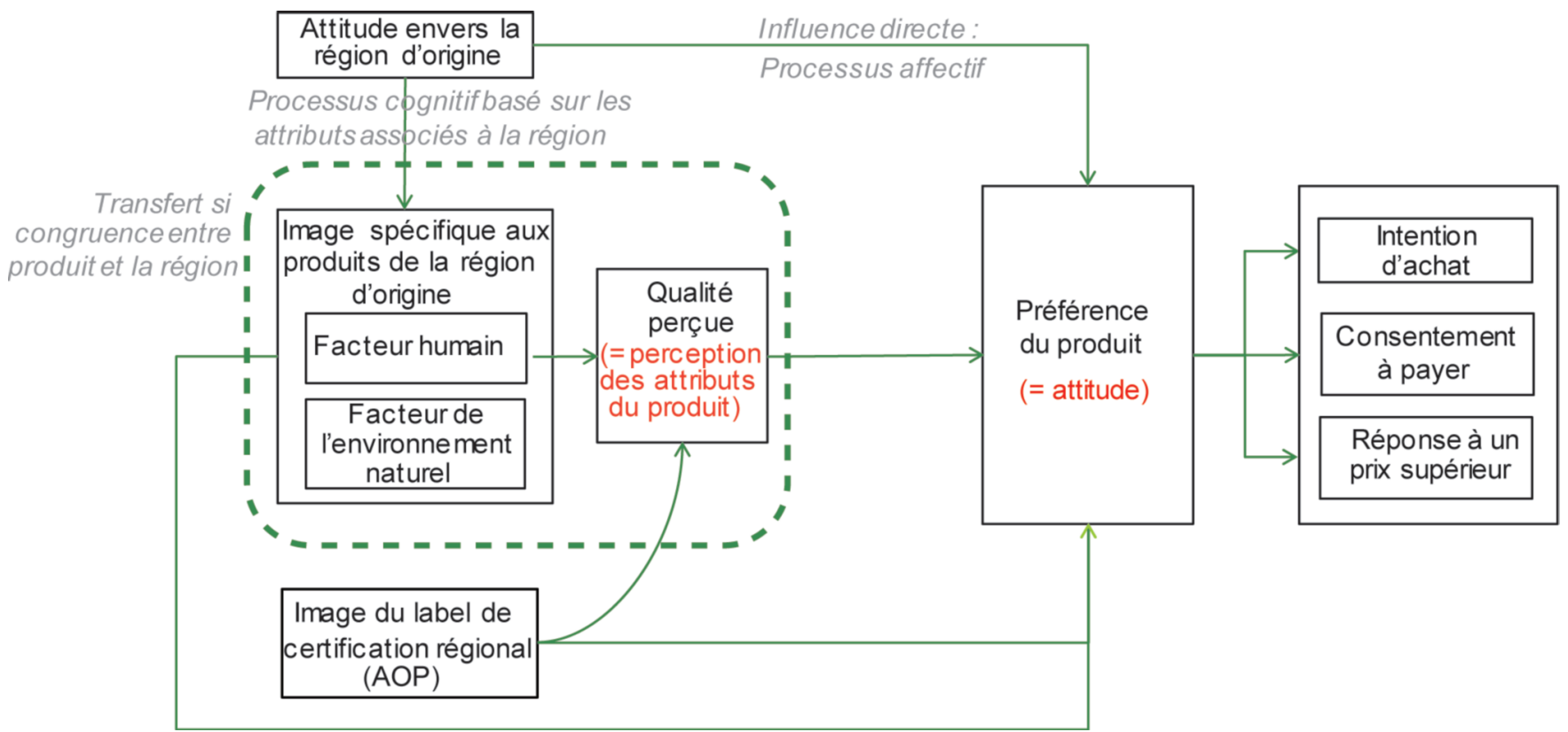

Ce schéma résume l'influence de la région d'origine (image et attitude) sur la préférence du produit. La préférence pour le produit est fonction de la perception des attributs du produit, elle-même significativement influencée par l'image régionale spécifique du produit et par l'attitude envers la région d'origine. L'image spécifique aux produits de la région d'origine est un construit à deux dimensions dont les composantes sont les facteurs humains et les facteurs d'environnement naturel. Son effet est principalement cognitif et spécifique au produit considéré (nécessité de congruence). L'attitude envers la région d'origine influence les préférences de manière directe à travers un processus affectif.

Ce modèle synthétise plusieurs données de littérature et reste à confirmer par des travaux complémentaires. II décrit un processus général sans pondération des variables. 
i) La composante cognitive. De manière indirecte, l'attitude influence l'image spécifique de la région qui, en cascade se répercute sur la perception des attributs, qui influence à son tour la préférence pour le produit régional selon un processus cognitif;

ii) la composante affective. De manière directe, l'attitude envers la région d'origine a une influence à travers un processus affectif. Ainsi, le nom d'un produit régional peut provoquer chez les consommateurs des émotions qui jouent un rôle important dans le comportement du consommateur (Holbrook et Hirschmann 1982). Ces émotions ont une influence sur la perception des attributs cognitifs du produit (Isen et al 1978);

iii) la composante conative (intentions comportementales).

Ces trois composantes de l'attitude envers la région d'origine interagissent entre elles : le concept-image pays et le concept d'affinité, qui traduisent respectivement les dimensions cognitive et affective sont tous les deux déterminants pour l'intention d'achat (dimension conative) (Bernard et Karoui 2013) et du consentement à payer des consommateurs pour des produits dont l'origine est protégée (Stefani et al 2006, van Ittersum et al 2007). Associée aux qualités du produit, l'image régionale peut créer une identité unique pour le produit et dans ce sens créer de la valeur ajoutée (Van Ittersum et al 2007). Le critère « origine régionale » ressort d'ailleurs comme la 1ère raison d'achat de produits AOC dans les études CREDOC (critère en progression de +7 points entre 2007 et 2010) devant les arguments « saveur», « fabrication artisanale », " sécurité », " taille des exploitations » et "santé » (Hebel 2010). Toutefois, Verlegh et Steenkamp (1999) soulèvent que le pays d'origine a un effet plus important sur la qualité perçue, plutôt que sur l'attitude vis-à-vis du produit ou sur l'intention d'achat.

L'effet du pays/région d'origine reste cependant encore mal compris (Aurier et al 2004), notamment parce que l'importance de l'effet pays d'origine, sa nature et son impact diffèrent en fonction de la présence de la marque, du prix, $d$ 'autres attributs extrinsèques et intrinsèques ou encore selon le contexte de l'évaluation (Aurier et Fort 2005). La marque en particulier peut avoir un impact fort sur le jugement de typicalité d'un produit de terroir. Plus la marque est typique des produits du pays ou de la région d'origine, plus l'association entre pays et marque est forte (Odou et Nicholson 2000). Il faut également souligner que des expériences défavorables ou incohérentes avec ce qui semble être un produit régional authentique (imitations) affaiblit l'attitude des consommateurs envers les produits authentiques (Van Ittersum et al 2007) avec pour conséquence une diminution du désir d'achat et du consentement à payer pour ce produit. Or, le champ de l'origine et des formes traditionnelles de production laisserait la place à des imitations ou des versions édulcorées des produits originaux (Grunert et Aachman 2016). La compétition injuste par les imitations décourage les producteurs réguliers de l'utilisation de l'origine et peut, au final affecter les économies rurales de ces territoires (Van Ittersum et al 2007).

\section{2 / Effet des labels ou signes d'identification de l'origine}

Les consommateurs semblent donner de l'importance à l'authenticité des produits alimentaires labellisés, ceux-ci présentant plus de transparence sur la production et également une promesse de produit final de meilleure qualité (Groves 2001). Aussi, des travaux ont cherché à comprendre comment l'attribut extrinsèque " indication géographique (AOP, IGP) » agit au niveau du consommateur et peut venir compléter la seule information d'origine du produit. Il est intéressant de noter que les dimensions perçues d'un signe d'identification de l'origine (l'AOP par exemple) diffèrent de celles perçues pour l'attribut « terroir ». En effet, Van Ittersum et al (2007) par ses travaux fait ressortir deux dimensions fondamentales de l'AOP aux yeux des consommateurs :

i) Une garantie de qualité (haute qualité, exclusivité) ;

ii) Un soutien économique (assurance que les AOP augmentent le revenu des producteurs, créent de l'emploi dans la région d'origine).

La dimension de garantie de qualité améliore la qualité perçue tandis que la dimension de soutien économique renforce leur attitude envers le produit sur la base du support de l'économie locale (Van der Lans 2001, Van Ittersum et al 2007). L'appréciation des consommateurs d'une protection de l'origine des produits régionaux est largement pilotée par la garantie que la protection apporte vis-à-vis de la qualité du produit. Cet effet sur la dimension de qualité garantie suggère que le logo AOP aide à améliorer la transparence du marché des produits régionaux de qualité, en permettant aux consommateurs de faire des meilleurs choix.

Toutefois, ces résultats sont à nuancer, certains travaux ayant montré que l'effet de l'AOP peut être limité à certaines catégories de consommateurs et que d'autres n'ont pas montré d'effet direct d'un logo AOP sur la préférence d'un produit (Van der Lans 2001) ni sur la considération de l'AOP comme un critère d'achat en tant que tel pour les consommateurs (Olivier et al 2014). Dans une revue de littérature, Grunert et Aachmann (2016) notent que le rôle des signes de qualité européens que sont les AOP, IGP et STG reste relativement faible dans la prise de décision des consommateurs : il peut avoir un rôle mais de manière moins importante que d'autres informations telles que la marque, l'origine. À noter que l'impact du signe de qualité est également variable selon le produit étudié, son lien à une origine régionale, la présence d'une marque plus ou moins forte, etc. Menapace et al (2009) montrent que le bénéfice supplémentaire pour les IGP et les AOP est relativement plus faible que le bénéfice pour le pays d'origine, conduisant certes à des plus-values mais de plus en plus faibles sur l'étiquetage de l'origine plus ce dernier est précis. Sur le consentement à payer un prix supérieur, les résultats sont partagés. En général, les études utilisant des entretiens directs ou des analyses conjointes confirment un consentement à payer supérieur pour des produits labélisés (Olivier et al 2014, Grunert et Aachmann 2016). Toutefois, ces résultats sont à nuancer. Bouamra-Mechemache (2014) montre via des modélisations économiques que les consommateurs sont plus sensibles aux variations de prix des fromages AOP que non-AOP. Deux autres études citées par Grunert et Aachmann (2016) étayent ce point de vue, montrant que les produits AOP ne sont pas moins sensibles au prix que les produits non AOP.

\section{3 / Facteurs de variation dans la perception des produits de terroir}

Par définition, la perception d'un bien ou d'une caractéristique par le consommateur est un processus subjectif. Afin de rendre compte de cette variabilité individuelle, les études ont généralement recours à deux types de descripteurs :

i) Les variables sociodémographiques : âge, sexe, niveau d'étude, revenu, etc. Par exemple, l'âge et le genre peuvent conduire assez fréquemment à des différences perceptuelles. La catégorie socio-professionnelle ou le revenu peuvent influencer le choix d'un type de marque (Lenglet 2011, Kreziak et al 2012). Si les panels ne font ressortir qu'un effet modéré du revenu sur la pénétration des produits laitiers AOP dans les foyers (indice 101 sur une base 100 pour les revenus aisés vs 97 pour les revenus modestes), l'effet âge est plus important avec une pénétration forte pour les couples d'âge moyen (106) et senior (107) et faible pour les jeunes célibataires (87) 
ou célibataires d'âge moyen (93). Les foyers sans enfant (104) sont également plus consommateurs que les autres structures familiales (INAO et CNAOL 2015).

ii) Les variables psychologiques : motivation, personnalité, valeurs, style de vie, etc. Moins fréquentes dans les études consommateurs menées par les filières fromagères de terroir, les caractéristiques psychologiques représenteraient pourtant d'après la littérature, des critères de segmentation plus pertinents que les variables sociodémographiques (Kreziak et al 2012). Ces éléments semblent confirmés par des travaux plus récents de Kreziak et Lenglet (2009) montrant que les variables sociodémographiques impactent peu les appréciations à l'égard des produits alimentaires. Plusieurs variables psychologiques sont listées dans la littérature :

- la recherche de variété dans son alimentation,

- l'implication,

- la confiance en soi,

- l'attitude à l'égard de l'alimentation (préoccupations de santé, éthiques, nouveauté).

Dans le cas des produits de terroir, deux autres descripteurs sont fréquemment utilisés de par leur importance dans la variation de la perception de ces mêmes produits :

i) Résidents vs non-résidents. La perception d'une image régionale peut différer selon que le consommateur réside dans cette région ou en est extérieur. Van der Lans (2001) note ainsi que l'information région d'origine a un effet direct sur la préférence envers un produit régional pour certains segments de consommateurs, en particulier parmi ceux qui vivent dans la région en question. Préférence qui ressort dans les panels de suivi des achats des ménages en fromages AOP avec des indices régionaux de manière quasi-systématique plus fort dans la région d'origine du produit que dans les autres régions (INAO et CNAOL 2015). Ce point a également été confirmé dans le projet IMAOP portant sur la perception des fromages AOP du MassifCentral par les consommateurs dans différents bassins de consommation (Olivier et al 2014). Van der Lans (2001) avance que cet effet « résidents » est dû essentiellement aux sentiments affectifs que ces consommateurs ont envers leur région d'origine ;

ii) la connaissance de la région. Plus globalement que le critère résident, c'est la connaissance de la région qui influence la perception du produit issu de cette région par le consommateur. Pour que le marketing sur cette origine soit réussi, deux conditions de base sont nécessaires : une proportion significative du marché ciblé informée de la région et des associations fortes et favorables entre les consommateurs et la région (Lenglet 2011). Ainsi, le nom de la région doit être connu pour être porteur, sans quoi il n'évoquera rien pour le consommateur. Plus les consommateurs ont des associations fortes et favorables à la région d'origine, plus l'attrait pour les produits de ces régions sera renforcé (Van der Lans 2001). Entre en jeu la connaissance de l'environnement de la région d'origine (si l'environnement est considéré comme sain, le produit sera perçu comme tel) mais également de son environnement immatériel (traditions, habitants, culture...) (Van der Lans 2001). Ces constats sont confirmés par les études consommateurs menées par les filières. Une étude de l'AFA (2010) réalisée sur un échantillon représentatif via 1'outil Prométhée de Kantar world Panel, montre ainsi que les consommateurs ont une plus forte propension à l'achat de fromages d'Auvergne lorsqu'ils se sont déjà rendus dans la région de production (indice variant de 116 pour le bleu d'Auvergne, le Cantal ou le Saint-Nectaire à 128 pour le Salers sur une base 100).

\section{4 / Application marketing : critères et leviers d'action}

\section{1 / Critères de différenciations et leviers d'actions}

Les différents travaux menés autour de la perception du terroir, de la région d'origine ou des AOP/IGP mettent en avant des variables d'actions marketing activables pour améliorer le jugement des consommateurs. Un préalable toutefois, les sources perçues du terroir par les consommateurs s'imposent à la stratégie marketing. De même, les attributs sensoriels et plus globalement tous les attributs intrinsèques sont généralement plus importants dans la prise de décision des consommateurs (Van Ittersum 2001) et s'imposent eux aussi à la stratégie marketing. Sur les attributs sensoriels, ceci est d'autant plus vrai que dans les cas des fromages AOP et IGP, les processus technologiques sont encadrés via les cahiers des charges. Il est par ailleurs important de rappeler que les propositions suivantes sur les variables d'action autour des facteurs «terroir » ou « origine » ne sont pas les seules à intervenir dans le choix d'un consommateur: les trois premiers critères d'évaluation d'un produit sont le prix, le goût, l'origine (Dekhili et D'Hauteville 2006). Dans le cas spécifique des fromages AOP du massif central (Olivier et al 2014) les principaux critères d'achat sont le goût
(80,1\% des consommateurs), le prix $(48,6 \%)$, le type de lait $(31,2 \%)$, l'aspect du fromage $(30,6 \%)$, l'origine géographique $(13,7 \%)$ et l'AOP $(8,5 \%$ des consommateurs).

Au niveau des opérateurs économiques, l'utilisation d'un signe de qualité tel que l'AOP ou l'IGP peut être un premier levier en protégeant les produis régionaux et ainsi en évitant les copies et les phénomènes de spoliation de réputation. L'appréciation des signes d'origine par les consommateurs peut aussi fournir des opportunités pour augmenter la demande de ce dernier en marquetant le produit régional avec un signe de protection de l'origine. L'utilisation d'une AOP ou IGP doit toutefois faire partie de stratégies marketing cohérentes (van der Lans et al 2001). L'effet de l'utilisation d'un de ces signes doit toutefois être modéré (cf. 4.2). Au niveau institutionnel, l'efficacité de la promotion de ces signes de qualité peut être améliorée en ciblant des messages différents selon les cibles. Par exemple, la dimension de soutien économique peut être plus pertinente pour les consommateurs qui ont des liens étroits avec la région, tandis que la dimension de garantie qualité sera pertinente pour les consommateurs en général (Van Ittersum et al 2007). Sur ce point, le même auteur signale qu'il est essentiel d'informer plus largement sur le sens et les garanties apportées par les AOP/IGP, de manière à ce que le consommateur puisse faire un choix informé entre ces produits protégés et d'autres produits de qualité. Il y a nécessité d'atteindre un vrai niveau de conscience/connaissance des systèmes de certification (SIQO) avant de pouvoir évaluer leur efficacité en termes d'objectifs commerciaux (Tregear et al 1998).

Par ailleurs, pour les produits de terroir, jouer sur la dimension « temps culture » qui regroupe à la fois l'histoire du produit et les rituels associés, pourrait être pertinent en termes de positionnement et de communication, cette dimension ayant un très fort pouvoir d'évocations positives (Aurier et Fort 2005, confirmé par Lenglet 2011). D'après ces trois auteurs, plus cette dimension est déterminante dans la perception des attributs du terroir, plus les attributs terroir du produit seront valorisés par les consommateurs. Dans ses travaux, Lenglet (2011) montre ainsi que d'un point de vue du consommateur, une communication sur l'organisation humaine (coopératives) dans le cas de l'Abondance laitier a un impact positif. Cette prise en compte de la dimension humaine semble intéressante tant pour favoriser le premier achat (impact sur les attentes) que les achats réguliers (amélioration du jugement global après consommation). Cette variable « temps 
et culture » peut être d'autant plus intéressante à activer que les filières tendent à insister plus sur la dimension origine du terroir alors que les consommateurs en valorisent surtout la dimension humaine. En effet, la dimension « origine » seule ne semble pas liée à la qualité perçue dans l'esprit des consommateurs (Aurier et al 2004). Plus largement, lier les produits aux «marqueurs culturels» ou aux images locales, telles que les traditions culturelles et le patrimoine, améliore la valeur du produit. De plus, il a été suggéré que l'association des produits alimentaires locaux avec le territoire présente le potentiel pour la localité de conserver une plus grande partie des avantages économiques, tout en maintenant un certain contrôle sur le type d'activité économique qui s'y produit (Skuras et Dimara 2004).

La dimension territoriale n'est pas non plus à négliger. Ainsi, dans ses travaux, Lenglet (2011) montre que d'un point de vue du consommateur, c'est une communication sur la dimension naturelle du terroir qui a l'impact le plus positif sur le fromage Abondance, qu'il soit laitier ou fermier.

Sur une étude quantitative appliquée au reblochon, Kreziak et Lenglet (2009) font ressortir que, d'un point de vue de la demande, communiquer sur les conditions locales de production contribue à valoriser le produit par rapport à son évaluation sur la base de ses seules qualités intrinsèques (apparence, couleur, forme, taille, structure...). En rentrant sur des critères de différenciation plus fins, le critère «petit producteur » et dans une moindre mesure le critère « environnement » sont plus efficaces pour valoriser les reblochons fermiers. Le critère agriculture de montagne est lui plus efficace pour valoriser le reblochon laitier. Les auteurs soulignent également qu'un positionnement sur ces critères nécessite de segmenter le marché, principalement sur des variables psychologiques qui se sont révélées plus pertinentes que les variables sociodémographiques. Ces propositions qui se placent du côté d'un marketing de la demande nécessitent de connaître les centres d'intérêts des consommateurs et surtout la nature des territoires qui les portent et les ressources qu'ils peuvent valoriser (Hirczak et al 2008). En ce sens, un rapprochement avec la valorisation des aménités d'un territoire (Kreziak et al 2012) ou avec le modèle du panier de biens (Hirczak et al 2008), est intéressant. Les ressources territoriales qui entourent un produit contribuent à le mettre en valeur. Les aménités environnementales, paysages, biodiversité, mais aussi patrimoine, architecture, histoire, culture et traditions, biens publics locaux, jouent le rôle d'une sorte d'écrin localisé
(Hirczak et al 2008). Les résultats du projet IMAOP (Olivier et al 2014) montrent l'importance pour les producteurs de créer avec les consommateurs diverses relations de proximité avec le territoire de production. Un effort particulier en communication ciblée sur toutes les composantes de l'attitude pourrait être fourni pour réussir à mieux exposer les liens au lieu.

\section{2 / Moyens d'activation et appro- che marketing globale}

La manière d'activer l'origine d'un produit peut également être un levier d'action. Ainsi dans ses travaux, Luomala (2007) démontre qu'activer l'origine nationale d'un produit par le biais cognitif favorise l'achat de produits étrangers, tandis qu'activer cette origine nationale par le biais affectif renforce la consommation de produits nationaux. Ainsi, des campagnes de communication sur le thème « acheter local» seraient plus efficaces en communiquant sur de l'affectif (sentiments, émotions) que sur une dimension cognitive (valeur prédictive et de confiance ; une activation cognitive entraîne un éloignement des consommateurs des produits d'origine). Par ailleurs, ces travaux suggèrent que plus l'aliment est transformé, moins la signification que le consommateur attache au pays d'origine est prononcée.

Plus globalement, les travaux de Fort et Fort (2006) soulignent les enjeux d'une approche marketing cohérente avec l'attente du consommateur : tant sur la performance du produit que sur la sauvegarde à long terme des sources de différenciations de l'ensemble de la catégorie terroir. Il est ainsi important pour les groupements de producteurs et opérateurs économiques des filières de terroir d'éviter les trop fortes dissonances entre l'image du produit et la démarche marketing telle que perçue par le consommateur. L'adéquation doit se faire de manière fortuite avec un produit «non apprêté ». Les travaux de Gallen (2005) sur le concept des représentations mentales soulignent que la dis-confirmation des attentes du consommateur sur les qualités organoleptiques ou sanitaires de l'aliment peut produire des conséquences néfastes sur le plan hédonique ou psychologique. Les travaux de Kreziak et Lenglet (2009), confirmés par des résultats du projet IMAOP (publication à venir), montrent qu'en matière de fromages, il est important de favoriser la première expérience de dégustation du consommateur avec le fromage, ce qui va favoriser par la suite l'acte d'achat. Au-delà du seul travail de positionnement et de promotion, l'exigence de qualité organoleptique et sanitaire doit donc faire l'objet d'une vigilance constante de la part des organisateurs de l'offre.
Enfin, sur l'origine, il paraît important de souligner que la marque à laquelle elle est associée constitue là aussi une variable d'action marketing. L'attitude pour l'origine jouant à l'identique pour tous les acteurs locaux, la congruence entre marque et origine reste un facteur très privé puisque certaines marques locales seront perçues comme étant plus congruentes que d'autres et bénéficieront plus de l'effet d'origine. Une PME avec une marque locale et perçue comme telle par les consommateurs aura ainsi plus de facilités à bénéficier de l'effet d'origine, capacité plus facilement mise en doute par les consommateurs pour des grandes marques (Aurier et Fort 2004). L'interaction de la marque avec le logo AOP est également variable. Là aussi l'effet de l'AOP semble moins fort sur une marque nationale connue et au contraire plus fort sur une marque de moindre notoriété. L'effet serait opposé par contre pour le logo IGP avec un consentement à payer plus fort pour une marque nationale adossée au logo IGP (Grunert et Aachmann 2016).

\section{3 / Prospective, ouverture, besoin en recherche}

Si les critères de différenciation et leviers d'action autour du marketing des produits de terroir ne manquent pas, de nombreuses pistes restent tout de même à explorer. En effet, la définition et la perception de ces produits est suffisamment large pour laisser la place à un ciblage et à une différenciation au sein des marchés de produits traditionnels (Vanhonacker et al 2010). Les travaux menés par Kreziak et Lenglet sur le reblochon ou sur l'Abondance en sont illustratifs. De même, l'effet de superposition des labels de qualité ou encore la cohabitation marque-label de qualité serait intéressant à développer.

L'importance d'une dimension telle que « temps et culture » souligne l'importance de l'exploration du champ de l'immatériel et ouvre des perspectives pour des travaux de recherche placés du côté de la demande. L'étude qualitative sur les usages et attitudes de consommation des fromages (Demarty 2010) fait ressortir ce besoin de réappropriation, de réinvention.

Enfin, s'il est montré que les logos AOP et IGP peuvent avoir un rôle éventuellement variable selon les produits et la marque, il serait intéressant de compléter l'étude de leur impact dans des situations d'achat réelles. En effet, la méthodologie des principales études sur ce champ, impliquant une exposition forcée aux informations du produit, induit un biais dans les réactions des consommateurs par rapport à des situations d'achat quotidiennes. D'autant que pour certains 
auteurs, la majorité des achats alimentaires nécessite une implication faible des consommateurs, même si plusieurs résultats nuancent ce propos. Cela semble notamment vrai sur les fromages, leur achat étant impliquant sur deux dimensions : intérêt et plaisir (projet IMAOP, publication à venir). Aussi, des études complémentaires sur l'implication, les processus d'attention et de perception de ces SIQO en situation réelle d'achat semblent indispensables.

\section{Conclusion}

Le principal objectif de cette revue de littérature était de préciser les mécanismes à l'œuvre dans la perception et l'attitude des consommateurs vis-à-vis des produits de terroir, avec un focus particulier sur les fromages de terroir issus de filières organisées.

La définition du concept « terroir » fait ressortir deux dimensions intimement liées : une dimension territoriale (facteurs naturels) et une dimension culturelle et historique (facteurs humains). Si l'on se place d'un point de vue consommateur, les sources perçues du terroir font ressortir trois dimensions :

i) métier (regroupant savoir-faire, recette et tradition);

ii) temps et culture (regroupant histoire et rituel);

iii) origine géographique (regroupant territoire, région et terre).
Ces dimensions sont ensuite développées à travers l'apport des études pays/région d'origine. L'importance de l'attribut extrinsèque « origine » sur la perception du produit par le consommateur est soulignée. Il affecte directement la valeur alimentation par son rôle symbolique, infère les croyances quant à la qualité du produit et se comporte comme un attribut essentiel de signalisation de la qualité.

La manière dont cette information agit sur le consommateur est détaillée et cette revue propose un schéma de synthèse (figure 1). La préférence pour le produit est fonction de la perception des attributs du produit, elle-même significativement influencée par l'image régionale spécifique du produit et par l'attitude envers la région d'origine. L'image spécifique aux produits de la région d'origine est un construit à deux dimensions dont les composantes sont les facteurs humains et les facteurs d'environnement naturel. Son effet est principalement cognitif et spécifique au produit considéré (nécessité de congruence). La présence d'une AOP ou d'une IGP peut venir compléter la seule information d'origine du produit mais son effet est relativement plus faible que le bénéfice pour l'origine. Dans toutes ces analyses, il convient de tenir compte d'un certain nombre de variables affectant la perception des produits par les consommateurs. Si les variables sociodémographiques ou encore des facteurs résidents/non-résidents et connaissance de la région sont déjà prises en compte dans les stratégies marketing des produits de terroir, d'autres telles les variables psychologiques pourraient aider à mieux segmenter les marchés.
Plusieurs pistes d'action marketing proposées dans la littérature sont présentées, avec la vigilance qu'elles ne pourront pas se substituer à des variables importantes dans l'évaluation des produits tels le prix, le goût, etc. Il ressort une nécessité de développer des démarches marketing cohérentes avec l'attente des consommateurs, qui ne soient pas en dissonance avec le produit. Au niveau des opérateurs et des organismes en charge des IG, les SIQO de type AOP/IGP doivent être intégrés de manière cohérente dans les stratégies marketing, de même que les marques dont la congruence avec l'origine peut engendrer des bénéfices substantiels. Marqueter le produit avec un signe d'identification de l'origine en insistant plus fortement sur la qualité et sur la dimension des bénéfices économiques du label, deux dimensions plus à même d'impacter la qualité perçue et donc indirectement le consentement à payer, pourrait s'avérer gagnant. La dimension « temps et culture » pourrait être plus utilisée pour son très fort pouvoir d'évocations positives, même si des études plus fines par produit pourraient être nécessaires pour mieux tirer parti des composantes les plus évocatrices (environnement, organisation humaine...). La manière d'activer l'origine d'un produit est également à prendre en compte : le levier affectif en jouant sur l'attitude envers la région d'origine semble particulièrement pertinent et serait plus efficace pour des messages « acheter local ». Évidemment, ces pistes n'ont de sens que s'il n'y a pas dis-confirmation des attentes du consommateur sur les qualités organoleptiques ou sanitaires des produits, d'où la nécessité d'une exigence de qualité constante de la part des organisateurs de l'offre.

\section{Références}

AFA, 2010. Les fromages d'Auvergne et les foyers français. Kantar Worldpanel.

AFP, 2014. Retour aux racines et au plaisir chez les consommateurs dans le monde en 2020. http://www.lafranceagricole.fr/actualites/ alimentation-retour-aux-racines-et-au-plaisirchez-les-consommateurs-dans-le-monde-en2020-etude-1,0,89101769.html

AFP, 2015. Les marques de distributeurs misent sur le terroir pour rattraper le terrain perdu.

Amilien V., 2005. « Préface : à propos de produits locaux ». Anthropology of food. http://aof. revues.org/306

Amilien V., Fort F., Ferras N., 2007. Hyper-real territories and urban markets: changing conventions for local food - case studies from France and Norway. Anthropol. Food, S2. http://aof. revues.org/446

Aubert N., 2006. L'individu hypermoderne. Éditions Erès, Toulouse, France, 2-6.
Aurier P., Fort F., 2005. Effets de la region d'origine, du produit, de la marque et de leurs congruences, sur l'évaluation des consommateurs: application aux produits agroalimentaires. Rech. Appl. Marketing, 20, 29-52.

Aurier P., Fort F., Sirieix L., 2004. Les produits de terroir du point de vue des consommateurs sources perçues et associations au terroir. In Actes du XX $\mathrm{X}^{\mathrm{èm}}$ Congrès de l'AFM.

Bérard L., Marchenay P., 1995. Lieux, temps et preuves. La construction sociale des produits de terroir. Terrain, 153-164.

Bérard L., Marchenay P., 2000. Le vivant, le culturel et le marchand : les produits de terroir. Autrement, 194, 191-216.

Bérard L., Marchenay P., 2004. Les produits de terroir, entre culture et règlements. CNRS Éditions, Paris, France, 229 p.

Bernard Y., Zarrouk-Karoui S., 2013. Renforcer les intentions d'achat et les consentements à payer grâce au sentiment d'affinité du consommateur envers un pays étranger. In: Actes du 29ème Congr. Int. AFM.

Beylier P., Messeghem K., Fort F., 2010. Les MDD du terroir au cœur de la légitimité territoriale des distributeurs. In : Biennale internationale de la négociation commerciale. 26.

Blair M.E., Innis D.E., 1996. The effects of product knowledge on the evaluation of warranteed brands. Psychol. Marketing, 13, 445-456.

Bouamra-Mechemache Z., Monier-Dilhan, S., Orozco V., 2014. Les AOC fromagères : quelle perception des consommateurs, quels atouts pour les producteurs? INRA Sci. Soc., 5, 4p. http://prodinra.inra.fr/?locale=frH! ConsultNoti ce: 285342

Bowen S., Mutersbaugh T., 2014. Local or localized? Exploring the contributions of FrancoMediterranean agrifood theory to alternative food research. Agric. Human Values, $13 \mathrm{p}$. https://s3.amazonaws.com/academia.edu. 
documents/36213628/Bowen and Mutersbau gh 2013.pdf?AWSAccessKeyId=-AKIAIWO WYYYGZ2Y53UL3A\&Expires $=1505803624 \&$ Signature $=x r 1 C M u c r 9 Q L y M Q B F V f x A X B v E$ Kls\%3D\&response-content-disposition $=$ inline $\% 3 \mathrm{~B} \% 20$ filename $\% 3 \mathrm{DB}$ owen_and_Muters baugh Foods Local or Loc.pdf

Brown S., Kozinets R.V., Sherry J.F., 2003. Teaching old brands new tricks: retro branding and the revival of brand meaning, J. Marketing, $67,19-33$.

Camus S., 2004. Proposition d'échelle de mesure de l'authenticité perçue d'un produit alimentaire. Rech. Appl. Marketing, 19, 39-63.

Casabianca F., Sylvander B., Béranger C., Coulon J.B., Roncin F., 2008. Terroir et Typicité : deux concepts-clés des Appellations d'Origine Contrôlée. Essai de définitions scientifiques et opérationnelles. In: Produits agricoles et alimentaires d'origine : enjeux et acquis scientifiques, 199-213. Presented at Colloque international de restitution des travaux de recherche sur les Indications et Appellations d'Origine Géographiques, Paris, France. http://prodinra.inra.fr/record/25920 In : Symp. Int. Territoires Enjeux Dev. Régional

Celsi R.L., Olson J.C., 1988. The role of involvement in attention and comprehension processes. J. Consumer Res., 15, 210-224.

Chamard C., Liquet J.C., 2009. L'évaluation de l'image perçue des régions françaises. Université de Pau et des Pays de l'Adour.

Chever T., Renault C., Renault S., Romieu V., 2012. Value of production of agricultural products and foodstuffs, wines, aromatised wines and spirits protected by a geographical indication (GI). Final report. $85 \mathrm{p}$.

CIRAD-Sar, 1996. Systèmes agroalimentaires localisés : organisations, innovations et développement local. Montpellier, France.

Darpy D., Guillard V., 2012. Comportements du consommateur. Éditions Dunod, Paris, France, $24 \mathrm{p}$.

Dhaene S., Theet C., 2010. Produits laitiers d'Appellation d'Origine de Normandie Synthèse et recommandations. Eurofins Marketing Research.

Dekhili S., D'Hauteville F., 2006. Place de l'origine dans la qualité et dimensions de l'image Perceptions des experts francais et tunisiens, cas de l'huile d'olive. UMR MOISA. http:// www1.montpellier.inra.fr/bartoli/moisa/bartoli/download/moisa2006 pdf/WP 2-2006.pdf

Delfosse C., 1991. Savoir scientifique et transformation de la production : L'exemple du fromage (1880-1950). Ethnozootechnie, 47, 107 116.

Delvalée J., 2015. Comment les consommateurs jugent l'offre locale. LSA, 19 février.

Demarty N., 2010. Usages \& attitudes de consommation de fromages. A\&S (Analyses et Stratégies) présenté à la journée d'études fromage, Maison du Lait, Paris, France.

Deselnicu O.C., Costanigro M., SouzaMonteiro D.M., McFadden D.T. 2013. A metaanalysis of geographical indication food valuation studies: what drives the premium for origin-based labels? J. Agric. Resour. Econ., 38, 204-219
Dubois B., Laurent G., Czellar S., 2001. Consumer rapport to luxury: Analyzing complex and ambivalent attitudes. HEC Paris.

Fischler C., 1990. L'Homnivore. Sur les fondamentaux de la biologie et de la philosophie. Éditions Odile Jacob, Paris, France, 416p.

Fort F., Fort F., 2006. Alternatives marketing pour les produits de terroir. Revue française de gestion, 145-159.

Fort F., Rastoin J.L., 2009. Marchés agroalimentaires, choix du consommateur et stratégies d'entreprises fondées sur le territoire : le modèle européen des indications géographiques. In: Les produits de terroir, les indications géographiques et le développement local durable des pays méditerranéens. 93-119.

Gallen C., 2005. Le rôle des représentations mentales dans le processus de choix, une approche pluridisciplinaire appliquée au cas des produits alimentaires. Rech. Appl. Marketing, $20,59-76$

Groves A.M., 2001. Authentic British food products: a review of consumer perceptions. International J. Consumer Studies, 25, 246-254.

Grunert K.G., Aachmann K., 2016. Consumer reactions to the use of EU quality labels on food products: A review of the literature. Food Control, 59, 178-187.

Guillaume M., 1980. La politique du patrimoine. Éditions Galilée.

Halawany-Darson R., 2010. Le traitement de l'information dans le processus de prise de décision du consommateur : le cas de la traçabilité des produits alimentaires. Thèse de doctorat d'université en Sciences de Gestion. Clermont-Ferrand : Université d'Auvergne, 474p.

Häubl G., Elrod T., 1999. The impact of congruity between brand name and country of production on consumers' product quality judgments. International J. Res. Marketing, 16, 199-215.

Hebel P., 2011. Opportunités et menaces des tendances de consommation. Journée d'études fromage, Maison du Lait, Paris, France.

Hirczak M., Moalla M., Mollard A., Pecqueur B. Rambonilaza M., Vollet D., 2008. Le modèle du panier de biens. Econ. Rurale, 55-70.

Holbrook M.B., Hirschman E.C., 1982. The Experiential Aspects of Consumption: Consume Fantasies, Feelings, and Fun. J. Consumer Res. 9, 132 .

Holt G., Amilien V., 2007. Introduction: from local food to localiser food, Anthropology of Food, 2, http://aof.revues.org/index405.html

INAO, 2014. Les produits sous signes officiels de la qualité et de l'origine - chiffres clés., S2, http://aof.revues.org/405S2

INAO, CNAOL, 2015. Chiffres clés 2014 des produits sous signes de la qualité et de l'origine - produits laitiers AOP.

Isen, A.M., Shalker T.E., Clark M., Karp L., 1978. Affect, Accessibility of Material in Memory, and Behavior: A Cognitive Loop? J. Personal. Social Psychol., 36, 1-12.

Jacoby J., Olson J.C., Haddock R.A., 1971 Price, brand name, and product composition characteristics as determinants of perceived quality. J. Appl. Psychol., 55, 570-579.
Kotler P., Dubois B., Manceau D., 2003. Marketing management. Pearson Education, Paris, France.

Kreziak D., Lenglet F., 2009. Critères de différenciation des produits alimentaires Alpes du Nord: Le cas du reblochon. Laboratoire IREGE Institut de management de l'Université de Savoie, p125.

Kreziak D., Lacroix A., Lenglet F., 2012. Les produits de terroir : vecteurs de valorisation des aménités ? Revue d'Économie Régionale \& Urbaine, 831 .

Lenglet F., 2011. Quels critères pertinents de différenciation pour les produits alimentaires de terroir ? In: Actes du congrès scientifique annuel de l'Association Française du Marketing. 18 .

Letablier M.T., Nicolas F., 1994. Genèse de la typicité. Sciences des aliments, 14, 541-556.

Luomala H.T., 2007. Exploring the role of food origin as a source of meanings for consumers and as a determinant of consumers' actual food choices. J. Business Res., 60, 122-129.

Menapace L., Colson G., Grebitus C., Facendola M., 2009. Consumer preferences for countryof-origin, geographical indication, and protected designation of origin labels. Working paper No. 09021

Nelson P., 1970. Information and Consumer Behavior. J. Polit. Econ., 78, 311-329.

Nicolas F., Rainsard F., 2005. Etude d'image sur les fromages au lait de brebis. BVA.

Ngoulma J., 2015. Consumers' willingness to pay for dairy products: what the studies say? A Meta-Analysis. 9èmes Journ. Rech. Sci. Sociales. Nancy, France. 24p.

Obermiller C., Spangenberg E., 1989. Exploring the Effects of Country of Origin Labels: An Information Processing Framework. Adv. Consumer Res., 16.

Odou P., Nicholson P., 2000. L'ensemble des produits d'un pays : une catégorie cognitive ? Working paper Università Ca'Foscari Venezia : $1-26$.

Olivier V., Pichon F., Amblard C., Ginon E., Halawany-Darson R., 2014. La tendance à la régionalisation des achats, peut-elle amener à un renouvellement marketing des produits alimentaires de terroir extrarégionaux ? Cas des fromages AOP du Massif Central. In: Territorial marketing. 11p.

Poulain J.P., 1993. Identité régionale et tourisme vert à l'heure de l'Europe. Tourismes.

Rastoin J.L., 2004. Quelle stratégie pour les produits de terroir dans un contexte de globalisation des marchés? In: Cafés-débats à Marciac.

Richardson P.S., Dick A.S., Jain A.K., 1994. Extrinsic and Intrinsic Cue Effects on Perceptions of Store Brand Quality. J. Marketing, 58, 28-36.

Rosenberg M.J., Hovland C.I, 1960. Cognitive, affective, and behavioral components of attitudes. Attitude organization and change: An analysis of consistency among attitude components, 3, 1-14.

Rosselin C., 1994. La matérialité de l'objet et l'approche dynamique-instrumentale. In : Le 
paradoxe de la marchandise authentique. Imaginaire et consommation de masse. Warnier J.P., Editions l'Harmattan, Paris, France, 145-170.

Salvador-Perignon M., 2011. « Les représentations mentales du mode de production des produits alimentaires (France) ». Anthropology of food. http://aof.revues.org/6888

Sherif M., Cantril H., 1947. The psychology of ego-involvements: Social attitudes and identifications. John Wiley \& Sons Inc, Hoboken, NJ, US, viii, 525pp.

Skuras D., Dimara E., 2004. Regional image and the consumption of regionally denominated products. Urban Stud., 41, 801-815.

Simonson I., 1989. Choice Based on Reasons: The case of attraction and compromise effects. J. Consum. Res., 16, 158-174.

Sirieix L., 1999. La consommation alimentaire : Problématiques, approches et voies de recherche. Rech. Appl. Marketing, 14, 41-58.

Steenkamp J.B.E.M., 1986. Perceived quality of food products and its relationship to consumer preferences: theory and measurement. J. Food Qual., 9, 373-373.

Steenkamp J.B.E.M, 1990. Conceptual mode of the quality perception process. J. Business Res., 21, 309-333.
Stefani G., Romano D., Cavicchi A., 2006. Consumer expectations, liking and willingness to pay for specialty foods: Do sensory characteristics tell the whole story? Food Qual Pref., 17, 53-62.

Steptoe A., Pollard T.M., Wardle J., 1995 Development of a measure of the motives underlying the selection of food: the food choice questionnaire. Appetite, 25, 267-284.

Taylor J.P., 2001. Authenticity and sincerity in tourism, Ann. Tourism Res., 28, 1, 7-26.

Thevenot G., 2007, «Prise en compte du naturel dans la consommation : clarification du concept de naturel et proposition d'une échelle de mesure de la sensibilité au naturel des consommateurs » In : Actes des 12 èmes Journ. Rech. Marketing de Bourgogne, Dijon, France.

Tregear A., Kuznesof S., Moxey A., 1998 Policy initiatives for regional foods: some insights from consumer research. Food Policy, 23, 383-394.

Van der Lans I.A., van Ittersum K., De Cicco A. Loseby M., 2001. The role of the region of origin and EU certificates of origin in consumer evaluation of food products. Eur. Rev. Agricult. Econ., 28, 451-477.

Van der Meulen H.S., 2007. A normative definition method for origin food products. Anthropology of food. http://aof.revues.org/406
Van Ittersum, K., 2001. The role of region of origin in consumer decision-making and choice, Ph.D. Thesis, Wageningen University and Research Centre, The Netherland

Van Ittersum K., Candel M.J.J.M., Meulenberg M.T.G., 2003. The influence of the image of a product's region of origin on product evaluation. J. Business Res., 56, 215-226.

Van Ittersum L., Meulenberg M., Van Trijp H., Candel M., 2007. Consumers' appreciation of regional certification labels: a pan-european study. J. Agricult. Econ., 58, 1-23.

Vanhonacker F., Verbeke W., Guerrero L., Claret A., Contel M., Scalvedi L., ŻakowskaBiemans S., Gutkowska K., Sulmont-Rossé C. Raude J., Granli B.S., Hersleth M., 2010. How European consumers define the concept of traditional food: evidence from a survey in six countries. Agribusiness, 26, 453-476.

Verdú Jover A.J., Lloréns Montes F.J., Fuentes Fuentes M.a.d.M., 2004. Measuring perceptions of quality in food products: the case of red wine. Food Qual. Pref., 15, 453-469.

Verlegh P.W.J., Steenkamp J.B.E.M., 1999. A review and meta-analysis of country-of-origin research. J. Econ. Psychol., 20, 521-546.

\section{Résumé}

L'origine des produits alimentaires et en particulier la référence au terroir est devenue le support d'une segmentation importante dans l'offre proposée aux consommateurs. Cet article propose une revue de la littérature précisant les mécanismes à l'œuvre dans la perception et l'attitude des consommateurs vis-à-vis des produits de terroir avec un focus particulier sur les fromages de terroir issus de filières organisées. Après rappel du cadre conceptuel, cette synthèse développe les sources perçues du terroir par le consommateur, l'apport de la région d'origine et leurs effets sur la qualité, l'attitude et la préférence du produit. Ceci est complété par l'analyse des effets des labels ainsi que les facteurs de variation pouvant impacter la perception. La préférence pour le produit est fonction de la perception des attributs du produit, elle-même significativement influencée par l'image régionale spécifique du produit et par l'attitude envers la région d'origine. L'image spécifique des produits de la région d'origine intègre deux dimensions dont les composantes sont les facteurs humains (temps-culture) et les facteurs d'environnement naturel. La combinaison de ces deux dimensions correspond à une notion de terroir. Son effet est principalement cognitif et spécifique au produit considéré (nécessité de congruence). L'attitude envers la région d'origine quant à elle, influence les préférences de manière directe à travers un processus affectif. Ces résultats sont à modérer selon les produits étudiés, leurs caractéristiques et au regard des différents consommateurs. Enfin cet article soulève des pistes d'actions marketing pour appuyer le développement de ces produits de manière cohérente avec les attentes des consommateurs.

\section{Abstract}

\section{Consumers' perception and attitude towards regional "terroir" products: focus on "terroir" cheeses}

Food products origin and specifically "terroir" (regional products) has become a powerful segmentation factor of foods offered to consumers. This article provides a literature review detailing the mechanisms that influence consumer perception and attitude towards "terroir" products with a focus on "terroir" cheeses from organized sectors. After recalling the conceptual framework, this synthesis develops the perceived sources of the "terroir" by the consumer, the contribution of the region of origin and their effects on food quality, on attitude and preference toward the product. This is supplemented by the study of the impact of labels and also the factors impacting perception. The preference for the product depends on the perception of product attributes, which itself is significantly influenced by a specific regional image of the product and the attitude towards the region of origin. The specific image of region of origin products integrates two dimensions whose components are the human factors (time-culture) and factors of the natural environment. The combination of these two dimensions corresponds to a notion of terroir. Its effect is primarily cognitive and specifically to the product (need of congruence) while attitudes toward region of origin have a direct influence on preferences through an affective process. These results vary according to the nature and attributes of the studied products, and they are closely linked to consumers' characteristics. Finally, this article suggests possible marketing actions to support the development of these products consistently with consumers' expectations.

P. ZINDY, R. HALAWANY-DARSON, A. HAUWUY., 2017. Perception et attitude des consommateurs vis-à-vis des produits de terroir : implications pour les fromages. INRA Prod. Anim., 30, 229-240. 